\title{
ABSCESSO EPIDURAL EM PACIENTE PORTADOR DE PIOMIOSITE TROPICAL: RELATO DE CASO E REVISÃO DA LITERATURA
}

\author{
EPIDURAL ABSCESS IN PATIENT WITH TROPICAL PYOMYOSITIS: \\ A CASE REPORT AND LITERATURE REVIEW
}

\section{ABSCESO EPIDURAL EN PACIENTE PORTADOR DE PIOMIOSITIS TROPICAL: RELATO DE CASO Y REVISIÓN DE LA LITERATURA}

Rodrigo Rezende ${ }^{1}$, Igor Machado Cardoso², Saulo Gomes de Oliveira ${ }^{3}$, José Lucas Batista Junior ${ }^{4}$, Chárbel Jacob Junior ${ }^{5}$

\begin{abstract}
A piomiosite é uma infecção muscular profunda subaguda que pode originar abscessos intramusculares únicos ou múltiplos. Está associada a infecções sistêmicas, diabetes mellitus, terapia imunossupressora, AIDS e mieloma múltiplo. Descrevemos um caso de piomiosite tropical com acometimento da coluna vertebral que evoluiu para abscesso epidural, em paciente com história pregressa de espondilite anquilosante em tratamento com medicações imunossupressoras e apresentou trauma direto na coxa direita previamente ao início dos sintomas. O objetivo deste trabalho é descrever o acometimento raro da coluna vertebral em pacientes com piomiosite tropical, com apenas um caso descrito na literatura mundial, destacando a necessidade dos métodos complementares na investigação diagnóstica para o adequado tratamento e as possíveis complicações dessa doença. Os pacientes imunossuprimidos com dores musculares subagudas devem ser investigados quanto à piomiosite tropical e o diagnóstico precoce é fundamental para o sucesso do tratamento.
\end{abstract}

Descritores: Piomiosite; Abscesso epidural; Imunossupressão; Infecções estafilocócicas.

\section{RESUMO}

The pyomyositis is a subacute deep muscle infection that can cause single or multiple intramuscular abscesses and is associated with systemic infections, diabetes mellitus, immunosuppressive therapy, AIDS and multiple myeloma. We describe a case of tropical pyomyositis with spinal involvement evolving to epidural abscess. The patient had a history of ankylosing spondylitis treated with immunosuppressive medications and presented direct injury in the right thigh prior to onset of symptoms. This article describes the unusual involvement of the spine in patients with tropical pyomyositis, with only one case reported in the world literature, highlighting the need for complementary methods of diagnostic investigation, for proper treatment and the possible complications of this disease. In conclusion, immunosuppressed patients with muscle pain should be investigated as to the tropical pyomyositis and the early diagnosis is crucial to the success of treatment.

Keywords: Pyomyositis; Epidural abscess; Immunosuppression; Staphylococcal infections.

\section{RESUMEN}

La piomiositis es una infección muscular profunda y subaguda que puede originar abscesos intramusculares únicos o múltiples. Se vincula a infecciones sistémicas, diabetes mellitus, terapia inmunosupresora, SIDA [AIDS] y mieloma múltiple. Describimos un caso de piomiositis tropical, con acometimiento de la columna vertebral, que evolucionó para absceso epidural, en paciente con historial anterior de espondilitis anquilosante en tratamiento con medicaciones inmunosupresoras y que presentó traumatismo derecho, en el muslo derecho, previamente al inicio de los síntomas. El objetivo de este trabajo es describir el acometimiento raro de la columna vertebral en pacientes con piomiositis tropical, con solamente un caso descrito en la literatura mundial, destacando la necesidad de los métodos complementarios, en la investigación de diagnóstico, para el apropiado tratamiento y las posibles complicaciones de esa enfermedad. Los pacientes inmunosuprimidos, con dolores musculares subagudos, deben ser investigados cuanto a la piomiositis tropical y el diagnóstico precoz es fundamental para el éxito del tratamiento.

Descriptores: Piomiositis; Absceso epidural; Inmunosupresión; Infecciones estafilocócicas.

\section{INTRODUÇÃO}

A piomiosite é uma infecção bacteriana aguda do músculo esquelético que geralmente é causada pelo Staphylococcus aureus. O acúmulo de pus inicialmente é intramuscular e não secundário a infecção adjacente da pele, tecidos moles ou osso. Clinicamente é caracterizado por febre, dor muscular localizada, rigidez e ede$\mathrm{ma}^{1,2}$. Classicamente, a piomiosite é uma infecção dos trópicos, ocorrendo preferencialmente em pacientes jovens do sexo masculino, saudáveis e ativos. Nos países temperados ocorre mais em pacientes imunocomprometidos e debilitados ${ }^{2}$. Pode ser chamada de piomiosite primária, miosite purulenta tropical, miosite bacteriana ou piomiosite tropical.

\section{RELATO DE CASO}

Paciente do sexo masculino, 48 anos de idade, pardo, comerciante, natural do Espírito Santo, portador de espondilite anquilosante, submetido à artroplastia total bilateral dos quadris há 15 anos, procurou o Serviço de Ortopedia e Traumatologia queixando-se de

1. Chefe do Grupo de Coluna Vertebral do Vila Velha Hospital e da Santa Casa de Misericórdia de Vitória - Vitória, ES, Brasil.

2. Chefe do Grupo de Coluna Vertebral do Hospital Meridional, Médico Assistente Grupo de Coluna Vertebral da Santa Casa de Misericórdia de Vitória - Vitória, ES, Brasil.

3. Residente do $2^{\circ}$ ano de Ortopedia eTraumatologia do Vila Velha Hospital - Vila Velha, ES, Brasil.

4. Médico Assistente do Hospital da Santa Casa de Vitória e do Hospital Meridional, ES, Brasil.

5. Médico Assistente do Hospital da Santa Casa de Vitória e do Vila Velha Hospital - Vila Velha, ES, Brasil.

Trabalho realizado no Serviço de Ortopedia eTraumatologia do Vila Velha Hospital, ES.

Correspondência: Rua Desembargador Augusto Botelho, n²09, apto 801, Praia da Costa, Vila Velha-ES. Brasil. CEP 29101-110. E-mail: rezenderodrigo@ hotmail.com 
dor de forte intensidade nas coxas com limitação para deambular mais de 10 metros, de inicio há 30 dias após trauma na coxa direita. Procurou atendimento médico onde foi medicado com antiinflamatório e relatado soltura das próteses dos quadris. Não apresentando melhora dos sintomas deu entrada no nosso serviço com impossibilidade completa de movimentar a articulação coxo-femoral bilateral, temperatura axilar de 37,5 graus, dispnéia e desidratado $(++\backslash++++)$, abdome globoso, ausência de sinais flogísticos em membros inferiores e exame neurovascular normal (Figuras 1 e 2).

Exames complementares: foi solicitado hemograma completo que apresentava hemoglobina de 9,5, leucocitose de 12.000, sem desvio à esquerda, radiografia da bacia sem sinais de soltura das próteses, radiografia do abdome sem alteração. Solicitado tomografia computadorizada do abdome, pelve e região de quadris sendo visibilizado presença de abscesso no músculo psoas, quadríceps esquerdo e glúteo direito (Figuras 3 e 4).

Durante a investigação diagnóstica foi solicitada ressonância magnética coluna lombar para investigação de discíte vertebral que justificaria o quadro inicial de psoíte, porém foi observado presença de volumoso abscesso epidural (Figura 5).

Também foram investigados infecção fúngica, HIV e brucelose com resultado dos exames negativos. Foram colhidas hemoculturas e submetido ao tratamento de urgência com drenagem cirúrgica do psoas e quadríceps esquerda e região glútea direita. Foi realizada coleta de material purulento para cultura e antibiograma e iniciado esquema antibiótico com vancomicina e ceftriaxona. Houve crescimento de Staphilococus aureos sensível a diversos antibióticos.
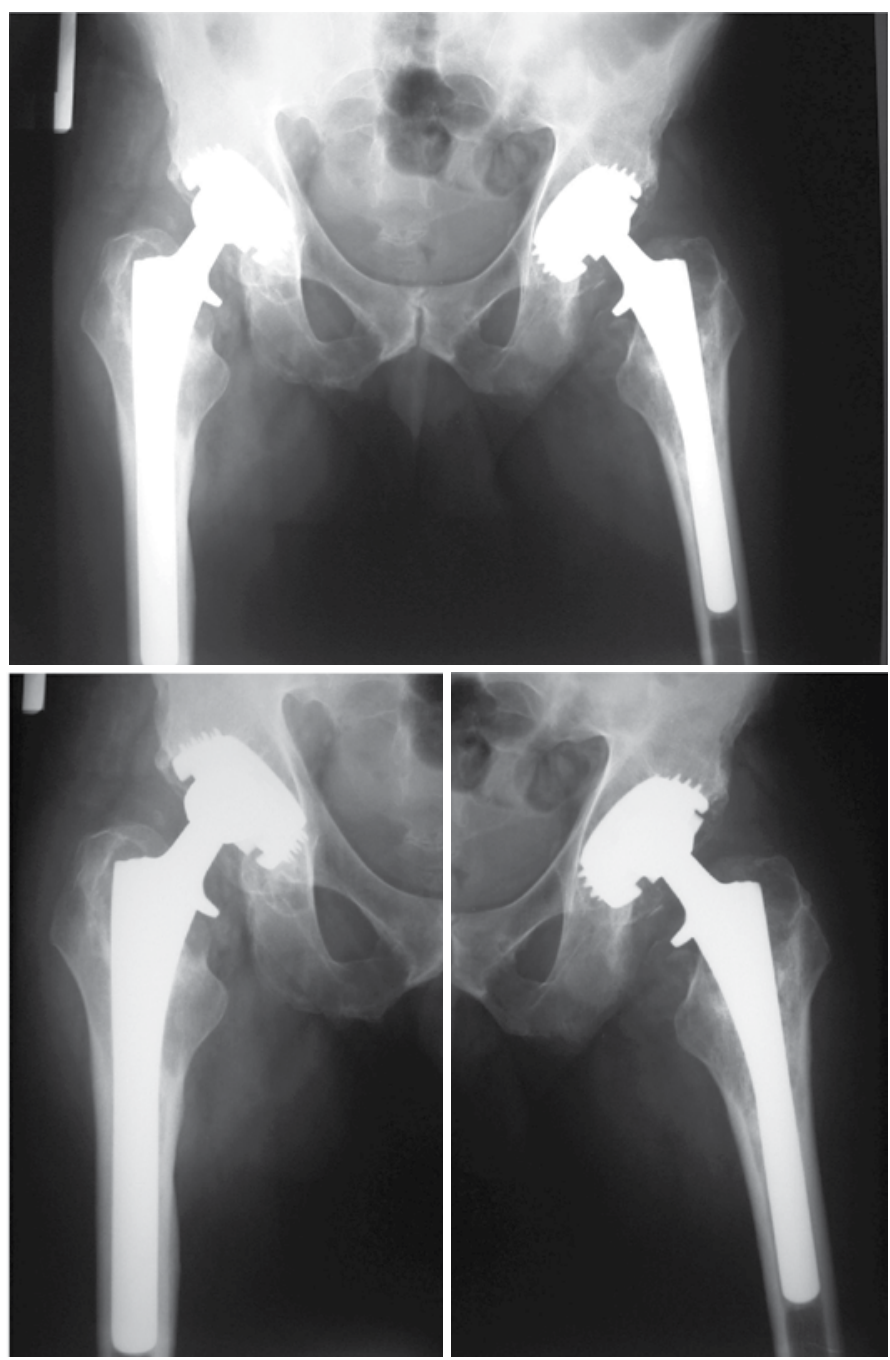

Figura 1. Radiografias bacia e quadris.

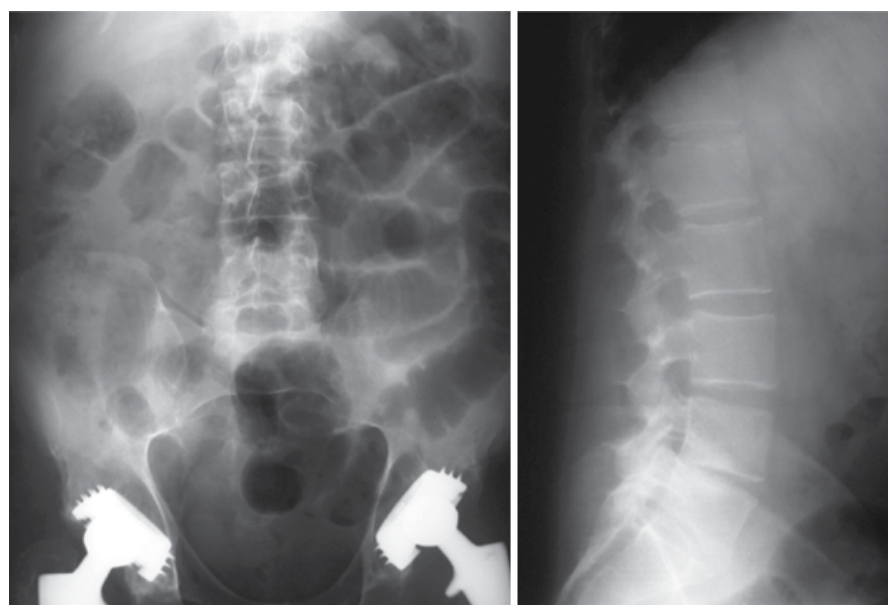

Figura 2. Radiografia de abdome frente e perfil de coluna - destaque de imagem no psoas esquerdo.

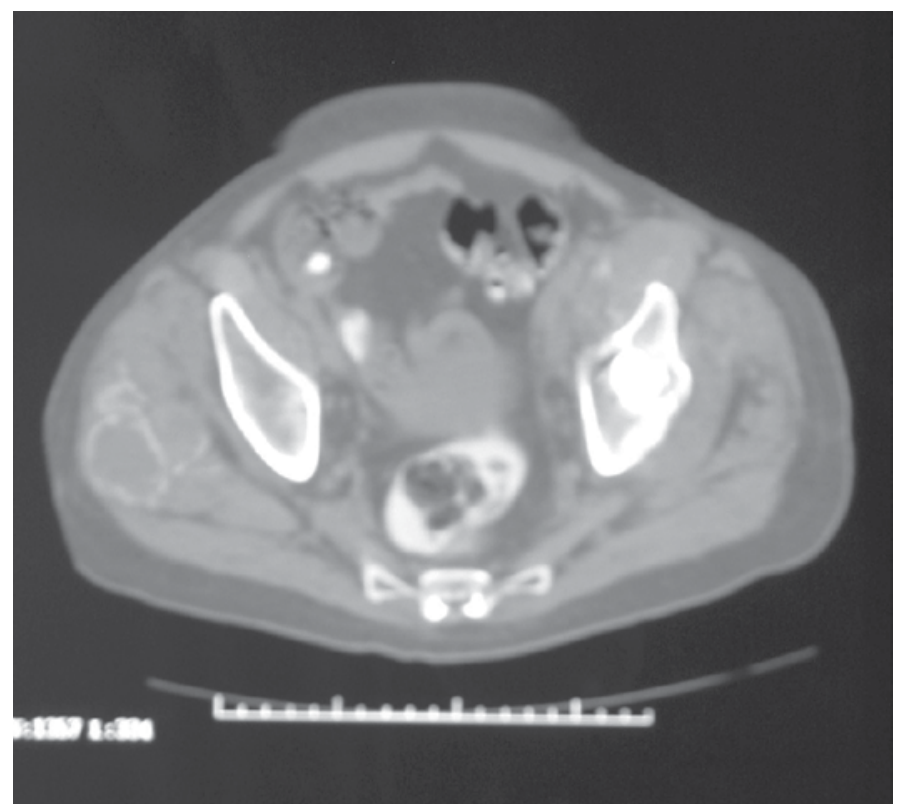

Figura 3. TC visibilizando abscesso glúteo direito.

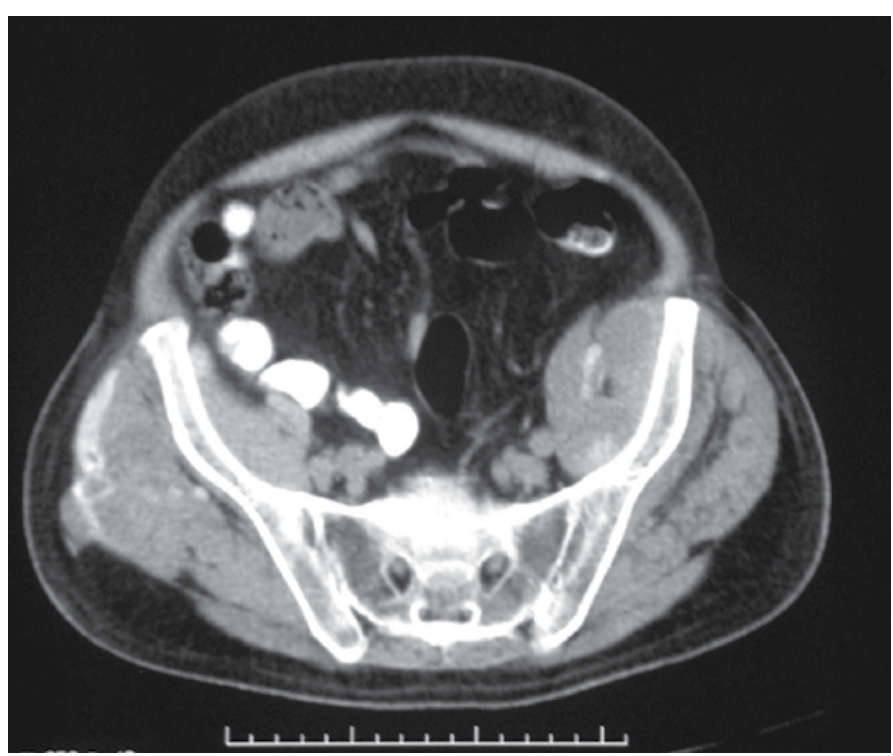

Figura 4. TC visibilizando psoíte esquerda. 
Após o resultado da cultura foi modificado o antibiótico para oxacilina 4 gramas endovenoso de quatro em quatro horas e foram necessário novas intervenções cirúrgica para limpeza cirúrgica e desbridamento dos abscessos. Como o paciente não apresentava nenhum sinal de compressão foraminal e de cauda equina foi optado pelo tratamento do abscesso epidural com antibioticoterapia endovenosa e acompanhamento radiológico semanal.

No final da quarta semana de antibioticoterapia com oxacilina não foi observado presença de abscesso epidural (Figura 6). O paciente apresentou alta hospitalar em seis semanas com melhora completa do quadro clínico.
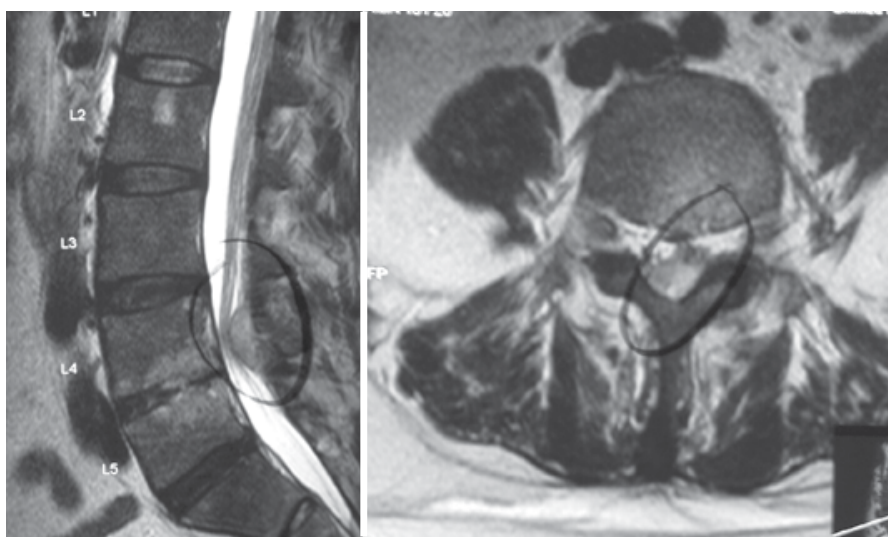

Figura 5. RNM: abscesso epidural nível L4-L5.
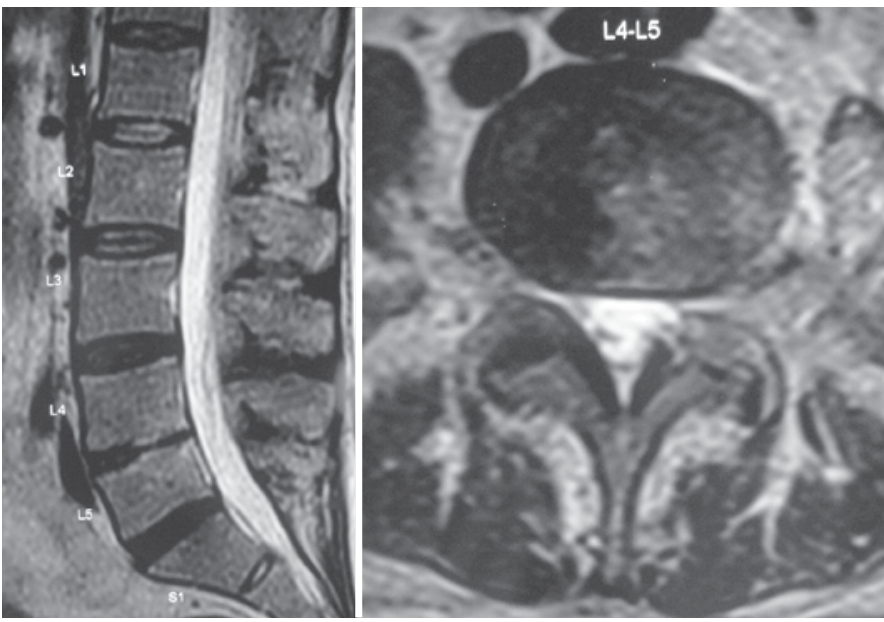

Figura 6. RNM: ausência abscesso epidural nível L4-L5.

\section{DISCUSSÃO}

A piomiosite (abscesso muscular primário) é uma infecção bacteriana do músculo que ocorre na ausência de sítio de infecção predisponente ${ }^{1}$. A maioria dos casos ocorre nos trópicos, sendo comumente chamada de piomiosite tropical. Em algumas regiões intertropicais da África, América Latina e ilhas do oceano Pacífico a doença é endêmica, correspondendo de 1\% a 4\% das admissões hospitalares ${ }^{3-8}$. Em 1971, Levin et al. ${ }^{4}$ relataram o primeiro caso em região temperada. Nos Estados Unidos a piomiosite é muito incomum, sendo descritos aproximadamente 100 casos entre 1970 e 1990, e na maioria das vezes os pacientes apresentavam antecedente de imigração de áreas tropicais. Drosos ${ }^{9}$ indica um possível aumento na incidência em áreas não tropicais e atribuem esse fenômeno ao melhor conhecimento da doença, ao aumento do número de pacientes imunocomprometidos e ao aperfeiçoamento das técnicas diagnósticas ${ }^{3,9,10}$.

Ocorre em todas as idades, sendo mais comum em crianças nas áreas tropicais e nos adultos e idosos em áreas temperadas, com preferência para o sexo masculino na proporção de 2:111 Aproximadamente $60 \%$ dos casos têm alguma condição predisponente como: diabetes mellitus, doença hepática, corticoterapia ou doença imunossupressora como leucemia, linfoma ou AIDS $^{1}$

O Staphylococcus aureus é responsável por 95\% dos casos nas áreas tropicais, porém nos Estados Unidos e outros países temperados é o agente causador em $66 \%$ a $70 \%$ dos $\operatorname{casos}^{12,13}$. Além do S. aureus outros microrganismos podem estar envolvidos, existem relatos de piomiosite causada por Streptocuccus pneumoniae ${ }^{14}$, Enterobactérias (Escherichia coli, Klebsiella pneumoniae, Salmonella spp.), Haemophilus influenzae, Neisseria gonorrhoeae, Anaeróbios (Fusobacterium nucleatum, Clostridium septicum) e Cryptococcus neoformans ${ }^{15}$. Também são descritos casos da doença causada por Candida, Aspergillus fumigatus e pelo bacilo da Tuberculose ${ }^{16,17}$.

A patogênese da piomiosite envolve previamente uma bacteremia, comumente assintomática e transitória. Em 20\% a 50\% dos casos há antecedente de traumatismo fechado ou exercícios vigorosos envolvendo a área. Estudos experimentais em laboratório produziram piomiosite com injeção intravenosa de $S$. aureus após traumatismo muscular em animais, sugerindo um fator local mecânico na patogênese ${ }^{1,18}$.

O quadro clínico geralmente envolve três estágios. No primeiro (ou estágio invasivo) o início é subagudo com febre, edema local com ou sem eritema, endurecimento da área e dor leve. Este período é geralmente negligenciado pelo paciente e na aspiração, se realizada, não há pus ${ }^{1}$

O segundo estágio (ou supurativo) ocorre após 10 a 21 dias ou mais e é o momento quando os pacientes são comumente diagnosticados. Aparecem sintomas como febre, edema em todo o músculo, calor e rubor, entretanto, o subcutâneo está preservado. Neste momento o pus pode ser aspirado do músculo ${ }^{1,3}$.

No terceiro estágio, manifestações sistêmicas de sepse e locais como eritema, dor acentuada e flutuação são encontradas ${ }^{1}$. Se não tratada ou ocorrer demora no diagnóstico e início do tratamento, a infecção pode progredir para abscesso metastático, choque séptico e falência renal como é observado em 10\% dos casos ${ }^{3}$. Ocasionalmente o quadro clínico é agudo, ao invés de subagudo, com mal-estar, febre alta e calafrios. Os abscessos musculares estão na maioria das vezes contidos por uma fáscia e o eritema local e calor pode ser mínimo até o processo se estender através do tecido subcutâneo após alguns dias ou semanas. Linfadenite regional não é característico ${ }^{1}$

Geralmente um único músculo está acometido, mas 40\% dos pacientes apresentam abscessos múltiplos. Os sítios mais comuns de acometimento são quadríceps femoral, glúteos e iliopsoas, mas pode haver envolvimento de diversos outros músculos ${ }^{5}$. O envolvimento dos músculos abdominais é incomum e a apresentação clínica pode mimetizar um abdômen agudo.

Dentre os exames laboratoriais observamos leucocitose e aumento de marcadores inflamatórios (VHS e proteína C reativa p. ex.). Eosinofilia é comum em pacientes com piomiosite tropical (mesmo na presença de leucocitose) e parece refletir a prevalência de infestação parasítica associada. Hemoculturas raramente são positivas ${ }^{3}$. Enzimas musculares podem estar elevadas, mas freqüentemente estão normais, apesar da extensão da destruição muscular ${ }^{1,3}$, entretanto, rabdomiólise com mioglobinúria e insuficiência renal foi relatada em um paciente com piomiosite ${ }^{19}$.

Durante o curso da piomiosite podemos suspeitar de diversos diagnósticos diferenciais como osteomielite, artrite séptica, apendicite ou diverticulite, distensão muscular, contusão ou hematoma, ruptura muscular e tromboflebite. A miosite do músculo iliopsoas pode mimetizar uma apendicite ou diverticulite, do músculo ilíaco mimetiza uma artrite séptica do quadril, e do músculo piriforme compromete o ciático simulando um abscesso epidural. A presença de crescimento lento, massa dolorosa em extremidade e febre baixa pode sugerir sarcoma.

A ultrassonografia é facilmente acessível e pode demonstrar um crescimento muscular no estágio inicial e a formação do abscesso no estágio supurativo da doença, através da presença de áreas 
hipoecóicas com ecos internos, especialmente se os sintomas estão localizados em extremidades ${ }^{20}$. A ressonância magnética avalia a presença de edema muscular focal e localiza a formação de abscessos com grande precisão. A tomografia computadorizada é uma alternativa no diagnóstico e na drenagem percutânea guiada dos abscessos, porém avalia com menos precisão os detalhes anatômicos nos estágios inflamatórios ${ }^{21}$.

A drenagem cirúrgica (aberta ou percutânea guiada por USG ou TC) de todos os abscessos é essencial. O início imediato de antibioticoterapia com administração intravenosa de penicilina beta-lactamase resistente como, por exemplo, a oxacilina ${ }^{22}$. O ajuste do antibiótico deve ser feito após o resultado da cultura e antibiograma da secreção drenada dos abscessos. A resposta à terapia é observada por exames laboratoriais como leucograma, VHS e PCR, diminuição da febre e exames de imagem seriados.

Marshman et al. ${ }^{23}$ descreveram recentemente o primeiro caso de abscesso epidural em um paciente com piomiosite tropical do sexo feminino de 56 anos de idade. Apresentamos neste trabalho o segundo caso descrito e o primeiro caso brasileiro apresentando abscesso epidural como complicação de piomiosite.

A maioria dos casos de abscessos epidurais ocorre em pacientes entre 30 e 60 anos de idade, o fator de risco mais comum é a diabetes mellitus, seguido de trauma, abuso de droga intravenosa, alcoolismo, AIDS, anestesia epidural e cirurgias espinhais. Anestesia ou analgesia epidural têm sido relatados como a causa em 5,5\% dos pacientes com abscesso epidural, sendo abscessos de pele e furúnculos os sítios de infecção mais comuns ${ }^{24}$.

Dos pacientes acometidos, $71 \%$ têm a dorsalgia como sintoma inicial e febre em $66 \%$. No segundo estágio ocorre irritação radicular seguido pelo terceiro estágio com início de déficits neurológicos, fraqueza muscular e incontinência urinária, bem como déficits sensitivos. Paralisia (quarto estágio) afeta apenas 34\% dos pacientes. O Staphylococcus aureus é agente mais comumente envolvido. A RM é de grande acurácia diagnóstica e é o método de escolha na propedêutica. Mielografia, largamente realizada no passado, não é mais recomendada. Punção lombar para determinação de concentração protéica no líquor não é necessária para o diagnóstico e há risco de inoculação bacteriana no espaço subaracnóideo com conseqüente meningite ${ }^{24}$.

A terapêutica de escolha é a descompressão cirúrgica por laminectomia (laminotomia pode ser tentada em crianças) combinada com antibióticos por quatro a seis semanas ${ }^{25}$. A mortalidade diminuiu de $34 \%$ na década de 50 para 15\% nos anos 90 . O problema essencial do abscesso epidural não é o tratamento, mas o diagnóstico precoce, antes dos sintomas neurológicos ocorrerem, com possível mielopatia irreversível ${ }^{24}$.

\section{REFERÊNCIAS}

1. Pasternack S, Swartz MN. Myositis. In: Mandell GM, Bennett JE, Dolin R, editors. Mandell, Bennett \& Dolin: Principles and Practice of Infectious Diseases. 6th ed. Philadelphia: Churchill Livingstone; 2005: 718-729.

2. Small LN, Ross JJ. Tropical and temperate pyomyositis. Infect Dis Clin North Am. 2005;19(4):981-9

3. Saïssy JM, Ducourau JP, Tchoua R, Diatta B. [Tropical myositis]. Med Trop (Mars). 1998;58(3):297-306.

4. Levin MJ, Gardner P, Waldvogel FA. An unusual infection due to staphylococcus aureus. N Engl J Med. 1971;284(4):196-8.

5. Bickels J, Ben-Sira L, Kessler A, Wientroub S. Primary pyomyositis. J Bone Joint Surg Am. 2002;84(12):2277-86

6. Horn CV, Master S. Pyomyositis tropicans in Uganda. East Afr Med J. 1968;45(7):463-71.

7. Sissolak D, Weir WR. Tropical pyomyositis. J Infect. 1994;29(2):121-7.

8. Eason R, Osbourne J, Ansford T, Stallman N, Forsyth JR. Tropical pyomyositis in the Solomon Islands: clinical and aetiological features. Trans R Soc Trop Med Hyg. 1989;83(2):275-8.

9. Drosos G. Pyomyositis. A literature review. Acta Orthop Belg. 2005:71(1):9-16.

10. Long SS, Pickering LK, Prober CG. Principles and practice of pediatric infectious diseases. 3rd ed. Philadelphia: Churchill Livingstone; 2008.

11. Chiedozi LC. Pyomyositis. Review of 205 cases in 112 patients. Am J Surg. 1979:137(2):255-9

12. Christin L, Sarosi GA. Pyomyositis in North America: case reports and review. Clin Infect Dis. 1992;15(4):668-77.

13. Harbarth SJ, Lew DP. Pyomyositis as a nontropical disease. Curr Clin Top Infect Dis. 1997:17:37-50

14. Collazos J, Fernández A, Martínez E, Mayo J, de la Viuda JM. Pneumococcal pyomyositis.

Case report, review of the literature, and comparison with classic pyomyositis caused by other bacteria. Arch Intern Med. 1996;156(13):1470-4.

15. Flagg SD, Chang YJ, Masuell CP, Natarajan S, Hermann G, Mendelson MH. Myositis resulting from disseminated cryptococcosis in a patient with hepatitis $\mathrm{C}$ cirrhosis. Clin Infect Dis. 2001;32(7):1104-7.

16. Bonomo RA, Graham R, Makley JT, Petersilge CA. Tuberculous pyomyositis: an unusual presentation of disseminated Mycobacterium tuberculosis infection. Clin Infect Dis. 1995;20(6):1576-7

17. Ahmed J, Homans J. Tuberculosis pyomyosits of the soleus muscle in a fifteen-year-old boy. Pediatr Infect Dis J. 2002;21(12):1169-71.

18. Miyake H. Beitrag zur Kenntniss des sogenannten Myositis infectiosa. Mitt Grenzgeb Med Chir. 1904;13:155

19. Armstrong JH. Tropical pyomyositis and myoglobinuria. Arch Intern Med. 1978;138(7):1145-6.

20. Quillin SP, McAlister WH. Rapidly progressive pyomyositis. Diagnosis by repeat sonography. J Ultrasound Med. 1991;10(3):181-4.

21. Gordon BA, Martinez S, Collins AJ. Pyomyositis: characteristics at CT and MR imaging. Radiology. 1995;197(1):279-86.

22. Chauhan S, Jain S, Varma S, Chauhan SS. Tropical pyomyositis (myositis tropicans): current perspective. Postgrad Med J. 2004:80(943):267-70.

23. Marshman LA, Bhatia CK, Krishna M, Friesem T. Primary erector spinae pyomyositis causing an epidural abscess: case report and literature review. Spine J. 2008;8(3):548-51.

24. Reihsaus E, Waldbaur H, Seeling W. Spinal epidural abscess: a meta-analysis of 915 patients. Neurosurg Rev. 2000;23(4):175-204.

25. Chao D, Nanda A. Spinal epidural abscess: a diagnostic challenge. Am Fam Physician. 2002;65(7):1341-6 